\title{
REVIEW
}

\section{Somatostatin receptor radionuclide therapy in neuroendocrine tumors}

\author{
Mintallah Haider1', Satya Das ${ }^{(12} 2$, Taymeyah Al-Toubah', Eleonora Pelle3 ${ }^{3}$ Ghassan El-Haddad4 and \\ Jonathan Strosberg(i) 1
}

'Moffitt Cancer Center, Department of GI Oncology, Tampa, Florida, USA

2Department of GI Oncology, Vanderbilt University, Nashville, Tennessee, USA

${ }^{3}$ Department of Oncology, University of Bari, Bari, Puglia, Italy

${ }^{4}$ Moffitt Cancer Center, Department of Diagnostic Imaging and Interventional Radiology, Tampa, Florida, USA

Correspondence should be addressed to J Strosberg: jonathan.strosberg@moffitt.org

\begin{abstract}
Peptide receptor radionuclide therapy (PRRT) using 177 Lu-DOTATATE has been approved for the treatment of gastroenteropancreatic NETs. An understanding of benefits and risks is important for the appropriate implementation of this therapy. This review summarizes study data supporting the use of radiolabeled somatostatin analogs for the treatment of advanced NETs and highlights risks, including potential toxicities in specific populations. Key ongoing clinical trials, including randomized studies, are designed to better define the position of PRRT within the broader therapeutic landscape. Preclinical and early-phase human studies are focused on the development of novel somatostatin-receptor agonists and antagonists, new radionuclides, and radiosensitizing combination therapies.
\end{abstract}

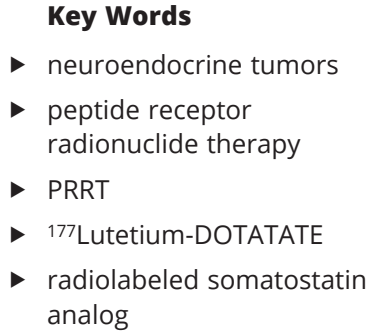

\section{Introduction}

Neuroendocrine tumors (NETs) encompass a heterogeneous family of neoplasms that can arise from various organ systems, with the gastrointestinal and pulmonary systems being the most common sites of origin (Dasari et al. 2017). The incidence of NETs has increased by nearly six-fold over the last three decades, and gastroenteropancreatic (GEP) NETs currently represent the second most prevalent gastrointestinal cancer after colorectal cancer (Dasari et al. 2017, Chauhan et al. 2019). NET biology is defined by grade and differentiation, with well-differentiated (WD) NETs typically demonstrating more indolent disease biology compared to poorly differentiated neuroendocrine carcinomas (Cambiaghi et al. 2017, Remes et al. 2019). An emerging class of therapeutics for patients with WD NETs consists of radiolabeled somatostatin analogs
(SSA), which fall under the broader therapeutic class of peptide receptor radionuclide therapy (PRRT).

PRRT enables the delivery of targeted radiation to cancer cells by binding a radionuclide to a peptide molecule, which localizes to a specific protein expressed on the cell surface. In the instance of radiolabeled SSAs, the peptide is a somatostatin receptor agonist that targets the somatostatin receptor (SSTR) (Fig. 1). SSTRs are G-protein coupled receptors that are over-expressed on the majority of WD NETs with subtype 2 (SSTR2) being the most frequently detected (Yamada et al. 1992, Oronsky et al. 2017). The characterization of SSTRs has contributed to the development of somatostatin receptor imaging (SRI) as well as PRRT. In this review, we will discuss the development of PRRT in WD NET patients, with a specific focus on efficacy and safety of the treatment modality as (c) 2021 Society for Endocrinology Published by Bioscientifica Ltd. Printed in Great Britain 

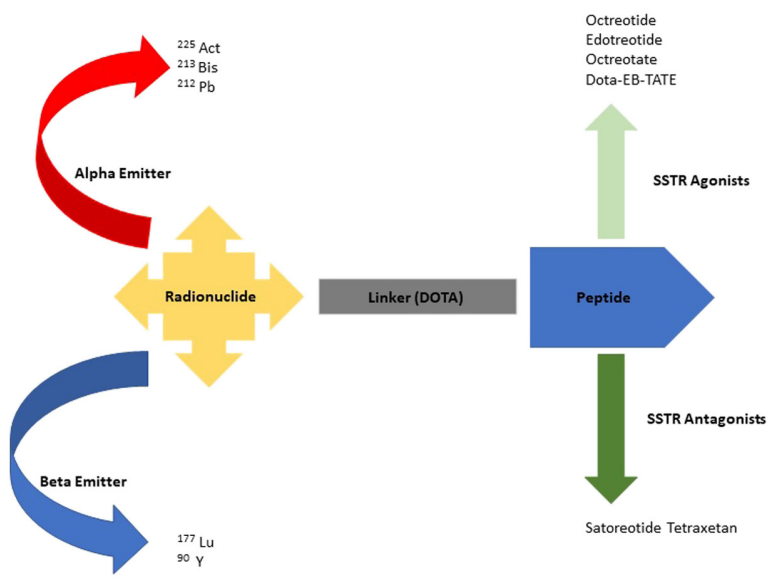

demonstrated in trials and cohort analyses in GEP NET and lung NET patients, ongoing questions about PRRT (optimal candidates, dosimetry, pretreatment imaging, biomarkers), and novel approaches (new combinations and agents, treatment sequencing) which are seeking to build on its promise.

\section{PRRT candidacy}

The European Neuroendocrine Tumor Society (ENETS) Consensus Guidelines for PRRT and the North American Neuroendocrine Tumor Society (NANETS) Consensus Statement on Patient Selection for PRRT define criteria for PRRT eligibility in patients (Hicks et al. 2017, Hope et al. 2020) Both ENETS and NANETS recommend that patients who are candidates for PRRT should have SSTR positive metastatic or inoperable NETs, possess an adequate hematologic reserve, have an adequate performance status, and exhibit either objective or clinical progression of disease/symptoms. The initial imaging modality used to confirm SSTR expression was indium-111 (111In) pentetreotide (OctreoScan). Although only formally validated for OctreoScans, SSTR expression is most commonly quantifiable through the Krenning score, which measures tumor SSTR expression on somatostatin receptor imaging (SRI) relative to healthy organs such as the liver and spleen (Krenning et al. 1989, Forbes et al. 2019). More recently, gallium-68 ( $\left.{ }^{68} \mathrm{Ga}\right)$-based somatostatin receptor imaging (i.e. ${ }^{68} \mathrm{Ga}$-DOTATATE) has demonstrated markedly higher sensitivity and improved image resolution (Coffey, 2019). PRRT is considered appropriate for patients having tumors with a Krenning's score of at least 2 (equivalent to the liver) on OctreoScan or SSTR expression higher than healthy liver on ${ }^{68} \mathrm{Ga}$-based SSTR PET (Hope et al. 2019). All lesions above the

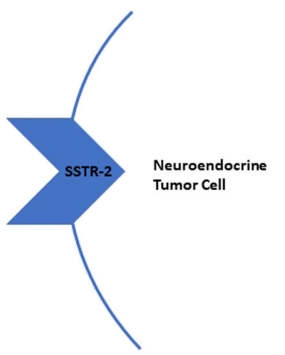

Figure 1

Schematic of peptide receptor radionuclide therapy.

sensitivity threshold for the scan (typically greater than approximately $8 \mathrm{~mm}$ in diameter) must express sufficient uptake. One study identified tumoral SUV uptake greater than twice the SUV uptake in the healthy liver as predictive of response to PRRT (Kratochwil et al. 2015).

There are several key points of difference between the ENETS and NANETS guidelines. The ENETS guidelines consider PRRT for well-differentiated grade 3 NETs and even NECs with SSTR-expressing disease, while NANETs guidelines point to the limited data with PRRT in these subgroups. A recent review discussed the results of four studies in which patients with G3 NENs received PRRT (Sorbye et al. 2020). The patient population consisted of primarily (50\%) well-differentiated, pancreatic primaries, with a Ki-67\%<55\%. Progression-free survival (PFS) was 19 months, 11 months, and 4 months for patients with well-differentiated G3 NETs, NETs with Ki-67<55\%, and poorly differentiated neuroendocrine carcinomas, respectively. More prospective data in this patient population is needed to further develop the guidelines, and the recently launched NETTER-2 (NCT03972488) trial aims to address this concern.

The two groups differ on the recommendation for required baseline renal function: ENETS more conservatively recommends that patients must have a creatinine clearance of $\geq 50 \mathrm{~mL} / \mathrm{min}$, while NANETS suggests that a creatinine clearance of $\geq 30 \mathrm{~mL} / \mathrm{min}$ is sufficient. Data suggest that renal toxicity with ${ }^{177} \mathrm{Lu}$-DOTATATE is negligible when prophylactic amino acids are used, even in patients with a glomerular filtration rate $<50 \mathrm{~mL} / \mathrm{min}$ (Strosberg et al. 2018b). Of note, typical formulations of amino acids used for PRRT contain $25 \mathrm{~g}$ of arginine and lysine mixed in $1000 \mathrm{~mL}$ of normal saline, while the NETTER-1 trial utilized commercial amino acids, such as those used for total parenteral nutrition, containing 19 amino acids. 
Another area of contrast between the two groups is in regard to treatment of patients with bulky mesenteric tumors, peritoneal carcinomatosis, and pretreated liver disease. NANETS guidelines discuss these patient subsets as potentially higher risk for PRRT while the ENETS guidelines do not address this. Underlying mesenteric and peritoneal disease is associated with chronic risk of bowel obstruction. Mesenteric metastases from small bowel NETs are often characterized by surrounding desmoplasia and theoretically, radiation to mesenteric or peritoneal disease may increase the risk of inflammationinduced obstruction. One study recently reported a bowel obstruction rate of $5 \%$ among patients with baseline mesenteric or peritoneal disease, including two occurrences of 'frozen abdomen' (Strosberg et al. 2020b). In another, bowel obstruction or ascites occurred in $28 \%$ of patients with diffuse peritoneal carcinomatosis treated with PRRT (Merola et al. 2020).

A small retrospective series found higher than expected rates of liver toxicity in a population of patients that had, on average, undergone significant prior liver embolization therapy (Riff et al. 2015). There are currently insufficient data to characterize the particular risk of PRRT before or after ${ }^{90} \mathrm{Y}$ radioembolization; however, logic dictates that patients who received high doses of bilobar intrahepatic ${ }^{90} \mathrm{Y}$ are at increased risk of radiation hepatitis if they receive PRRT in the setting of high burden liver disease. De novo burden of liver disease before PRRT initiation does not appear to be associated with an increased risk of liver toxicity with PRRT, however liver failure is possible in patients with massive tumor bulk (Strosberg et al. 2020a).

\section{Therapeutic radionuclides}

An ${ }^{111}$ In-labeled somatostatin analog, ${ }^{111}$ In-pentetreotide, was developed for use in scintigraphy in 1994 (Krenning et al. 1994). The initial PRRT experience was reported with high dose ${ }^{111}$ In-pentetreotide, the same radionuclide used in OctreoScan imaging (Valkema et al. 2002). Emission of Auger electrons accounts for the weak cytotoxic effects of high dose ${ }^{111} \mathrm{In}$. In this study, 50 patients underwent treatment with the agent, and 40 patients were eligible for assessment after cumulative doses ranging from 20 giga-becquerel $(\mathrm{GBq})$ to $160 \mathrm{GBq}$. Of the patients who were assessed for response, 1 (2.5\%) experienced a partial response (PR), and $20(50 \%)$ experienced stable disease (SD). Specific rates of myelosuppression were not reported; however, 3 of 6 patients who received more than $100 \mathrm{GBq}$ of therapy developed myelodysplastic syndrome or acute leukemia. Thus, $100 \mathrm{GBq}$ was deemed to be the maximum recommended dose for ${ }^{111}$ In-octreotide. No significant nephrotoxicity was reported. The limitation of this drug is its minimal cytoreductive capacity, likely due to the short-range of action and limited tissue penetration of Auger electrons (Sabet et al. 2016). Therefore, the theoretical benefit of $\beta$-emitting isotopes to elicit greater cytoreduction in NETs prompted their clinical development.

The initial experience with the $\beta$-emitting radionuclide Yttrium-90 $\left({ }^{90} \mathrm{Y}\right)$ was reported in a 29 patient NET cohort of mixed primary tumor types (Otte et al. 1999). Each of the analyzed patients received four or more doses of ${ }^{90}$ Y-DOTATOC every 6 weeks (cumulative dose of $6.1 \pm 1.3 \mathrm{GBq}$ ). Of the treated patients, 15 received amino acid infusions to prevent nephrotoxicity while the remainder did not. Twenty (69\%) of patients achieved SD, while six (21\%) achieved PR. Concerning toxicity, 24 (83\%) of patients did not demonstrate grade 2 or greater hematologic or renal toxicity. Of the five patients who did, all received $>7.4 \mathrm{GBq}$ of ${ }^{90}$ Y-DOTATOC and did not receive any amino acid infusions; four patients developed nephrotoxicity (two requiring dialysis) while two patients developed grade 3 anemia.

In another large cohort analysis of 1109 NET patients, with 25 different NET subtypes included, treated with ${ }^{90}$ Y-DOTATOC, treatment, and safety outcomes were reported (Imhof et al. 2011). Of the treated patients, $34.1 \%$ experienced any degree of treatment response, $5.2 \%$ experienced SD, and $60.5 \%$ demonstrated clinical response. Patients who achieved any treatment response experienced an improved overall survival (OS) compared to those who did not (44.7 months vs 18.3 months $(P<0.001)$. The toxicities experienced most commonly were transient grade $3 / 4$ myelosuppression $(12.8 \%)$ and permanent grade $4 / 5$ nephrotoxicity (9.2\%); one patient each developed myelodysplastic syndrome and acute myeloid leukemia. In another large 807 patient cohort analysis where patients were treated with ${ }^{90} \mathrm{Y}$-DOTATOC, lutetium-177 $\left({ }^{177} \mathrm{Lu}\right)$-DOTATATE or a combination of the two drugs (typically two cycles of each), 1.5\% of patients experienced grade 3/4 nephrotoxicity. Grade 3/4 nephrotoxicity rates with ${ }^{90} \mathrm{Y}$ alone, ${ }^{177} \mathrm{Lu}$ alone and with the combination were 2.8, 0 and 1.3\%, respectively (Bodei et al. 2015). Rates of nephrotoxicity are substantially higher with ${ }^{90} \mathrm{Y}$ compared to ${ }^{177} \mathrm{Lu}$, owing to the longer particle range of the former isotope (12 $\mathrm{mm}$ vs $2 \mathrm{~mm}$ ) (Bushnell et al. 2010, Imhof et al. 2011, Vinjamuri et al. 2013). Given its safety profile, ${ }^{177} \mathrm{Lu}$ became the lead betaemitting radionuclide utilized for PRRT. 
In one of the initial studies with ${ }^{177} \mathrm{Lu}$-DOTATATE, 35 patients with unresectable GEP NET tumors were treated (Kwekkeboom et al. 2003). Treatment doses were 3.7 GBq in 7 patients, $5.6 \mathrm{GBq}$ in 14 patients and $7.4 \mathrm{GBq}$ in 14 patients; 30 patients (86\%) received a total dose between 22.2-29.6 GBq. On initial response assessment, $37 \%$ achieved an objective response while $41 \%$ achieved SD. Grade 3 thrombocytopenia and leukopenia occurred in $4 \%$ and $1 \%$ of patients, respectively. In the initial US experience with ${ }^{177} \mathrm{Lu}$-DOTATATE, 37 patients with unresectable GEP NETs were treated up to four cycles (Delpassand et al. 2014). Twenty-two patients were assessable for response; $28 \%$ of patients experienced an objective response and SD was achieved in $41 \%$ of patients. The median progression-free survival (PFS) for treated patients was 16.1 months. Grade 2 and Grade 3 hematologic toxicity was experienced by $9.4 \%$ and $12.5 \%$ of patients, respectively. Grade 3 hepatotoxicity was experienced by $9.4 \%$ of patients.

The NETTER-1 study was the first randomized phase III study of ${ }^{177} \mathrm{Lu}$-DOTATATE and evaluated 231 patients with midgut (jejunal and ileocecal) NETs who had progressed on standard doses of octreotide LAR. Patients were randomized to receive four treatments of ${ }^{177}$ Lu-DOTATATE at a dose of $7.4 \mathrm{GBq}$ every 8 weeks in combination with standard-dose octreotide or high-dose octreotide $(60 \mathrm{mg}$ ) alone every 4 weeks (Strosberg et al. 2017). Median PFS was 8.4 months in the high-dose octreotide arm and was not reached in the PRRT arm (hazard ratio (HR) for disease progression or death 0.21 , 95\% CI, 0.13-0.33; $P<0.001)$. Response rates were 3\% in the high-dose octreotide arm and $18 \%$ in the PRRT arm $(P<0.001)$. In this analysis, grade $3 / 4$ neutropenia, thrombocytopenia and lymphopenia were reported in 1, 2 and $9 \%$ of patients in the PRRT arm, respectively. No patients treated with ${ }^{177} \mathrm{Lu}$-DOTATATE experienced treatment-related nephrotoxicity. In the first update from the study, median OS in the high-dose octreotide arm was 27.4 months and still not reached in the PRRT arm. In addition, quality of life measures demonstrated prolonged time to decline in global health status, physical functioning, role functioning, as well as disease specific symptoms including fatigue, diarrhea and pain with PRRT (Strosberg et al. 2018a). Of note, patients with higher liver tumor burden did not derive the same benefit in terms of PFS (Strosberg et al. 2020a).

In a large cohort analysis, 610 Dutch patients with midgut, foregut, hindgut and unknown primary NETs received treatment with ${ }^{177} \mathrm{Lu}$-DOTATATE (Brabander et al. 2017). For the safety analysis and efficacy analysis, 582 and
443 patients had data available, respectively. In patients included in the efficacy analysis, defined as those who received at least 3 cycles of ${ }^{177} \mathrm{Lu}$-DOTATATE, the median PFS was 28 months, median time-to-progression (TTP) was 36 months and median OS was 63 months. The objective response rate was 39\% while SD was achieved in 43\% of patients. For the subgroup of patients with progressive disease at baseline (54\% of efficacy cohort), median PFS was 30 months, median TTP was 36 months and median OS was 58 months. Grade 3/4 myelosuppression was experienced by $10 \%$ of patients; $5 \%$ experienced grade $3 / 4$ thrombocytopenia, $5 \%$ experienced grade $3 / 4$ leukopenia while $4 \%$ experienced grade 3 anemia. Myelodysplastic syndrome and acute leukemia occurred in $1.5 \%$ and $0.7 \%$ of patients, respectively. Only $0.4 \%$ of patients experienced grade 3/4 nephrotoxicity. Based on findings from the NETTER-1 study as well as this cohort analysis, ${ }^{177} \mathrm{Lu}$-DOTATATE obtained regulatory approval for progressive GEP-NET patients with SSTR-expressing disease.

A large retrospective analysis from Germany reported clinical outcomes and safety data from 1,048 NET patients treated with ${ }^{90}$ Y- and/or 177Lu-based PRRT (Baum et al. 2018). Overall, median PFS was 19 months (16.9-21) and median OS was 51 months (47.0-54.9). Patients who received a combination of both radionuclides, however, experienced augmented PFS and OS compared to monotherapy. Patients treated with ${ }^{177} \mathrm{Lu}$-DOTATATE monotherapy experienced longer OS than patients treated with ${ }^{90}$ Y-DOTATOC. Treatment related grade 3/4 adverse events were reported in less than $1 \%$ of patients. Dialysis was necessary in $0.4 \%$ of patients after treatment. Myelodysplastic syndrome and leukemia occurred in $2 \%$ of patients during follow-up. Tandem radionuclide administration appears promising and merits further evaluation, particularly in patients with bulkier disease burden, who may benefit from the enhanced cytoreduction with ${ }^{90}$ Y-based PRRT.

\section{Biomarkers}

Several studies have begun to evaluate whether there are any predictive factors for the success of PRRT. A retrospective analysis of 74 patients with welldifferentiated GEP NETs treated with PRRT showed that Ki-67 index had a significant impact on OS (Ezziddin et al. 2014). At a median follow-up of 82 months, median OS was 49 months for patients with grade 2 tumors, and not reached for patients with grade 1 tumors. Among https://erc bioscientifica com

https://doi.org/10.1530/ERC-20-0360 (c) 2021 Society for Endocrinology Published by Bioscientifica Ltd. Printed in Great Britain 
patients with grade 2 tumors, those with a lower ki- $67 \%$ (3-10\%) did better than those with ki-67\% in the higher range (15-20\%). Other factors found to be significant predictive factors were baseline tumor burden, Karnofsky performance score, and neuron-specific enolase level.

Investigators have also explored whether genomic signatures can identify the NET patients who may benefit optimally from PRRT with ${ }^{177} \mathrm{Lu}$-DOTATATE. A blood and tumor-based NET transcript assay (PRRT Predictive Quotient (PPQ)) has been developed and validated in 178 GEP NET and bronchial NET patients from three institutional cohorts (Bodei et al. 2018). The blood transcripts portion of the PPQ includes eight genes responsible for NET growth and metabolism while the tumor portion of the assay includes $\mathrm{Ki}-67 \%$. The target gene expression was quantified in a binary fashion using a cutoff of $\geq 5.9$ while the Ki- $67 \%$ was classified as high or low based upon a Ki-67 cut-off of $20 \%$ (or designation of atypical vs typical carcinoid in bronchial NET patients). The PPQ was developed in a 54-patient Italian cohort and was validated in German and Dutch cohorts. In the developmental cohort, PPQ was accurate in $94 \%$ of patients with a sensitivity of $100 \%$ and negative predictive value of $100 \%$ for PFS. PPQ positive patients had a median PFS which was not reached while PPQ negative patients had a median PFS of 8 months after completing PRRT. Similar sensitivity and negative predictive value were demonstrated in the validation cohorts.

Tumor avidity on fluorodeoxyglucose (FDG) PET/CT scans has also been demonstrated to be a poor prognostic factor for patients being considered for PRRT and is particularly relevant for patients with G3 NETs (Zhang et al. 2019). In a study of 55 patients with G3 NETs who received treatment with PRRT (with either ${ }^{177} \mathrm{Lu}$-DOTATATE or ${ }^{90}$ Y-DOTATOC) monotherapy or in combination with capecitabine, patients with higher FDG avidity on baseline scans (scores 3/4) had median PFS and OS times of 7.1 and 17.2 months, respectively. Patients with lower FDG avidity on baseline FDG PET/CT scans (scores 0-2) had median PFS and OS times of 24.3 and 42 months, respectively. Some investigators have proposed that all G3 NET patients should receive dual tracer imaging with FDG PET scans and ${ }^{68} \mathrm{Ga}$-DOTATATE PET scans to guide optimal management to ensure that all lesions express somatostatin receptors.

\section{Lung NETs}

The use of PRRT in lung NETs is limited to phase II studies and single institution experiences. In the Dutch cohort analysis referenced above, patients with lung NETs demonstrated a median PFS of 20 months (compared to 30 months with pancreatic and midgut NETs) (Brabander et al. 2017). In the German analysis cited above, median PFS in lung NET patients was 11 months compared to 20 and 22 months in patients with pancreatic and small-intestinal NETs, respectively (Baum et al. 2018). In aggregate, data for lung NET patients treated with PRRT appear to show inferior outcomes than GEP NET patents treated with PRRT. This may be because lung NETs express SSTRs less uniformly compared to GEP NETs (Kanakis et al. 2015).

In another recent analysis which aggregated several prospective studies of lung NET patients treated with ${ }^{177}$ Lu-DOTATATE, median PFS ranged from 15.7 to 29 months (Naraev et al. 2019). The median OS in these patients ranged from 35 to 48.6 months. Unsurprisingly, patients with typical lung NETs seem to have improved outcomes over atypical lung NETs after treatment with PRRT. Although regulatory approval has not been obtained in lung NET patients treated with PRRT, the experiences of large patient cohorts suggest a role for ${ }^{177} \mathrm{Lu}$-DOTATATE in patients with this disease.

\section{Sequencing of treatments}

Several studies are beginning to address the crucial question of where PRRT best fits in the sequence of therapies for NET patients. The NETTER-2 trial is exploring the efficacy of ${ }^{177} \mathrm{Lu}$-DOTATATE in treatment naïve grade 2 (Ki-67 > 10\%) and grade 3 GEP NET patients (NCT03972488). In this study, 222 patients will be randomized to ${ }^{177} \mathrm{Lu}$-DOTATATE or high-dose octreotide $(60 \mathrm{mg})$. The primary endpoint of the study is PFS. The COMPETE trial is randomizing progressive GEP NET patients to ${ }^{177} \mathrm{Lu}$-DOTATOC vs everolimus (NCT03049189). The primary endpoint of this 300 patient study is PFS. The OCCLURANDOM trial is randomizing progressive WD pancreatic NET patients to ${ }^{177} \mathrm{Lu}$-DOTATATE or sunitinib (NCT02230176). The primary endpoint of this 80 patient randomized phase II study is 12-month PFS.

\section{Novel approaches}

Some of the most promising strategies to augment the efficacy of PRRT, among others, include intraarterial administration for liver-dominant disease, retreatment 
upon disease progression, addition of radiosensitizers and development of next-generation radiopeptides.

\section{Intraarterial treatment}

PRRT infused into the hepatic artery is designed to increase exposure to liver metastases while decreasing systemic toxicity. A retrospective analysis was performed in 55 patients who received 77 applications of intraarterial PRRT with ${ }^{177} \mathrm{Lu}$ (7.2 GBq) or ${ }^{90} \mathrm{Y}$ (4.2 GBq) (Singh et al. 2019). The primary endpoints of the analysis were PFS and OS. Of the patients included in the analysis, $85.4 \%$ possessed GEP NETs and $70.9 \%$ had liver-only disease. Across the entire cohort, combined PFS and OS were 29 months and 70 months. For patients with liver-limited disease, median PFS and OS were 33.4 and 75.8 months, respectively. An ongoing multicenter parallel assignment randomized study is comparing intraarterial ${ }^{177} \mathrm{Lu}$-DOTATATE to i.v. ${ }^{177} \mathrm{Lu}$-DOTATATE in well differentiated NET patients with liver-limited metastatic disease (NCT03590119).

\section{Liver-directed therapy}

HEPAR PluS, a phase 2 non-randomized trial, evaluated the addition of Holmium-166 (166-Ho) radioembolization to liver metastases within 20 weeks after four cycles of PRRT. The primary endpoint was objective liver tumor response in the treated liver volume, defined as either complete or partial response per RECIST 1.1, at 3 months post treatment. Thirty patients were evaluable for primary endpoint assessment: 13 patients (43\%) achieved an objective response. Most commonly reported grade 3-4 toxicities at 6 months post intervention included abdominal pain (10\%), elevated GGT (54\%) and lymphocytopenia (23\%) (Braat et al. 2018). One patient died due to radioembolization-induced liver disease; no other treatment-related SAEs.

\section{PRRT retreatment}

In patients who derive benefit from initial PRRT and develop disease progression, retreatment is an option, although the safety profile of this strategy is not yet fully defined. Retreatment with ${ }^{177} \mathrm{Lu}$-DOTATATE was recently evaluated in a cohort of 168 patients with advanced, progressive NETs who had previously undergone PRRT with a PFS of at least 18 months (van der Zwan et al. 2019). Patients who received a second treatment course had a median PFS of 14.6 months (95\% CI 12.4-16.9). While there was no grade $3 / 4$ nephrotoxicity reported, grade $3 / 4$ myelosuppression occurred in up to $7.7 \%$ of patients, with a $2.2 \%$ incidence of acute myeloid leukemia and myelodysplastic syndrome. Although more data with PRRT retreatment needs to be reported, the stable rate of myelodysplasia and acute myeloid leukemia was reassuring.

\section{Combination therapies}

The combination of capecitabine and temozolomide (CAPTEM) has demonstrated substantial activity in pancreatic NETs, and more modest activity in other primary sites. Several studies have evaluated this cytotoxic regimen in combination with PRRT (van Essen et al. 2008, Claringbold et al. 2012, Kesavan et al. 2014, Ballal et al. 2017, Yordanova et al. 2019). In one phase I/II clinical trial, 35 patients with advanced well-differentiated NETs were treated with four cycles of ${ }^{177} \mathrm{Lu}$-DOTATATE in combination with CAPTEM (Claringbold et al. 2012). Median PFS was 31 months (95\% CI 22-55) and median OS had not yet been reached with $90 \%$ of patients alive at the 24-month data cut-off. Patients experienced an ORR of $57 \%$ and a DCR of $94 \%$. Treatment was well tolerated, with one instance of grade 3 nausea and one instance of grade 3 neutropenia.

Another study reported safety data from a cohort of 65 patients with metastatic GEP NETs treated with four cycles of ${ }^{177} \mathrm{Lu}$-DOTATATE in combination with either capecitabine alone $(n=28)$ or CAPTEM $(n=37)$ (Kesavan et al. 2014). One of the 28 patients treated with capecitabine and PRRT developed short-term grade 3/4 anemia and one patient developed anemia and thrombocytopenia several years after treatment. Of the patients who received combination CAPTEM along with PRRT, eight patients experienced myelosuppression: one developed significant thrombocytopenia, one developed neutropenia, four patients developed anemia and two patients developed myelodysplastic syndrome. Based upon these study findings, a randomized phase II study with parallel arms in pancreatic NET and midgut NET patients exploring the combination of CAPTEM plus ${ }^{177} \mathrm{Lu}$-DOTATATE is ongoing (NCT02358356); preliminary findings from the midgut group suggest no difference in PFS or response rate benefit with the combination compared to ${ }^{177} \mathrm{Lu}$-DOTATATE monotherapy at the cost of increased toxicity (Pavlakis et al. 2020).

A phase I/II study of everolimus in combination with ${ }^{177} \mathrm{Lu}-\mathrm{DOTATOC}$ was recently published (Aljubran et al. 2019). Patients received four doses of PRRT concurrently with everolimus in the trial; maximum tolerated dose 
(MTD) of the mTOR inhibitor was determined to be 10 mg. The reported response rate was only $9 \%$. After a median follow-up of 18.9 months, median PFS in the treated cohort was 23.3 months. A total of $36 \%$ of patients experienced grade 3 toxicities including infection, fatigue, pneumonitis, and neutropenia while no patients experienced grade 4 toxicities. Based on the modest cytoreduction elicited by the treatment combination, along with the toxicity profile, it is unclear whether this combination is promising enough to be further developed.

Treatment approaches with tandem radiopeptides have also been evaluated in NET patients, with the theoretical advantage of utilizing radionuclides with variable penetration ranges (de Jong et al. 2005, Pach et al. 2012, Seregni et al. 2014, Kunikowska et al. 2017). In the initial prospective cohort study, 50 patients with foregut, midgut and hindgut NETs were divided equally into two groups (Group A and Group B) (Kunikowska et al. 2011). Group A patients received PRRT treatment with ${ }^{90}$ Y-DOTATATE while Group B patients received PRRT with a 1:1 mixture of ${ }^{177} \mathrm{Lu}-/{ }^{90} \mathrm{Y}$-DOTATATE. Median OS in Group A patients was 26.2 months while was not reached in Group B patients $(P<0.027)$. Though differences in tumor regression did not meet statistical significance, Group B patients demonstrated greater tumor regression. In a later study, 59 patients with metastatic midgut, foregut and hindgut NETs received PRRT with a 1:1 mixture of ${ }^{177} \mathrm{Lu}-{ }^{90} \mathrm{Y}$-DOTATATE forthree to five cycles. Disease control rate was $89 \%$ and at a median follow up of 75.8 months, patients experienced a PFS of 32.2 months and OS of 82 months. Safety profile was favorable with one incidence of grade 3 nephrotoxicity and one instance of myelodysplastic syndrome. It remains to be seen whether tandem radiopeptide PRRT may be utilized in future clinical trials in NET patients specifically with bulky disease.

Other approaches to increase the cytotoxicity of PRRT include combining the modality with possible radiosensitizers such as cellular signaling inhibitors, DNA damage inducers and DNA damage repair inhibitors (Spetz et al. 2017, Hofving et al. 2018, Purohit et al. 2018).

The hedgehog cellular signaling pathway is dysregulated in multiple NET subtypes, making it an attractive drug target. Hedgehog inhibitors as monotherapy have demonstrated anti-tumor effect in NET models and investigators have examined combining it with ${ }^{177} \mathrm{Lu}$-DOTATATE in GOT1-bearing BALB/C nude mice. Mice were treated with either sonidegib $(80 \mathrm{mg} / \mathrm{kg}$ twice weekly administered orally), a single injection of $0.03 \mathrm{GBq}{ }^{177} \mathrm{Lu}$-DOTATATE IV or a combination of both. Mice treated with a combination of sonidegib and ${ }^{177} \mathrm{Lu}$-DOTATATE demonstrated increased tumor regression and had longer time to progression compared to mice treated with either monotherapy. On pharmacodynamic studies, differential pathway activation (Wnt/B-catenin, PI3K/AKT/mTOR) was observed in tumors of mice treated with the combination therapy (Spetz et al. 2017).

HSP90 inhibitors increase DNA damage and apoptosis in treated cancer cells and have demonstrated preclinical promise in NET models both in vitro and in vivo. In BON1 xenograft models, mice treated with onalespib on days 1-4 (30 mg/kg i.p. administration) and ${ }^{177} \mathrm{Lu}$-DOTATATE on days 2-4 (.0037 GBq IV), demonstrated improved tumor growth slowing, survival and tumor regression compared to monotherapy with either agent. Specifically, complete responses were increased from $8 \%$ in xenografts treated with ${ }^{177} \mathrm{Lu}$-DOTATATE monotherapy to $29 \%$ in xenografts treated with onalespib and ${ }^{177} \mathrm{Lu}$-DOTATATE (Lundsten et al. 2020).

Poly(ADP-ribose)polymerase inhibitors (PARPi), which interfere with base excision DNA repair, have been combined with ${ }^{177} \mathrm{Lu}$-DOTATATE in NET models. Investigators published results from experiments of ${ }^{177} \mathrm{Lu}$-DOTATATE in combination with PARPi in BON-1 (GEP NET) and NCI-H727 (lung NET) cell lines. In BON-1 monolayer experiments, cell viability was markedly decreased in cells treated with the combination therapy compared to those treated with either therapy alone. This was confirmed in BON-1 spheroid models with proliferation assays. Results of these experiments were recapitulated in the NCI-H727 cell lines. The cell-line studies confirmed that PARPi augmented the DNA damage elicited by ${ }^{177} \mathrm{Lu}$-DOTATATE by inhibiting repair mechanisms and increasing double-strand DNA breaks (Purohit et al. 2018). Another study evaluated olaparib in combination with PRRT in SSTR2-expressing human osteosarcoma cells (U2OS) and rat pancreatic Ca20948 cells. PARP inhibition resulted in sensitization to 177-Lu-DOTATATE, with reduction in cell numbers and replication. There was an increase in double stranded breaks with combination therapy resulting in cell death. Notably, the addition of PARP inhibition had effect on proliferating cells only, without increased toxicity in other cells (Nonnekens et al. 2016).

These examples include just several of the compounds being combined with PRRT, including those with the strongest published preclinical rationale. Ongoing combination studies with PRRT are summarized in Table 1. 
Table 1 Ongoing combination studies with PRRT.

\begin{tabular}{|c|c|c|c|c|}
\hline Study & Phase & Treatment & Patient population & Outcomes \\
\hline NCT04029428 & II & 177LU-DOTATATE \pm 90Y-DOTATATE & $\begin{array}{l}\text { G1-G3 GEP NETs, lung NETs, } \\
\text { pheochromocytoma and } \\
\text { paraganglioma, NET-CUP } \\
\text { - with SSTR } \\
\text { overexpression }\end{array}$ & $\begin{array}{l}\text { PFS (primary) and OS/PS/CRS/HOS/ORR/ } \\
\text { safety assessments (secondary) }\end{array}$ \\
\hline NCT04086485 & $|/ I|$ & 177Lu-DOTATATE + Olaparib & $\begin{array}{l}\text { Inoperable or metastatic } \\
\text { GEP NETs }\end{array}$ & $\begin{array}{l}\text { MTD (primary, phase I) and ORR (primary, } \\
\text { phase II) }\end{array}$ \\
\hline NCT02736500 & $\mid / I I$ & $\begin{array}{l}{ }^{177} \text { Lu-DOTATATE + Metronomic } \\
\text { Capecitabine }\end{array}$ & $\begin{array}{l}\text { Inoperable or metastatic } \\
\text { GEP NETs }\end{array}$ & $\begin{array}{l}\text { Toxicity rate (primary, phase 1) ORR } \\
\text { (primary, phase II) and PFS (secondary, } \\
\text { phase II) }\end{array}$ \\
\hline NCT03325816 & $\mid / I I$ & 177Lu-DOTATATE + Nivolumab & $\begin{array}{l}\text { Extensive-stage small cell } \\
\text { lung cancer }\end{array}$ & $\begin{array}{l}\text { RP2D (primary, phase I), PFS (primary, } \\
\text { phase II) and OS/DCR/ORR/safety profile } \\
\text { (secondary, phase II) }\end{array}$ \\
\hline NCT03457948 & II & $\begin{array}{l}\text { 177Lu-DOTATATE or liver directed } \\
\text { therapies + Pembrolizumab }\end{array}$ & G1-G3 NETs & $\begin{array}{l}\text { ORR (primary), DOR/safety profile/PFS/ } \\
\text { iPFS (secondary) }\end{array}$ \\
\hline NCT04194125 & II & 90Y-DOTATOC + CAPTEM & G1-G2 GEP NETs & $\begin{array}{l}\text { PFS (primary), OS/PS/CRS/HOS/ORR/ } \\
\text { safety profile (secondary) }\end{array}$ \\
\hline NCT03044977 & I & 90Y-DOTATOC + 131|-MIBG & $\begin{array}{l}\text { NETs with SSTR } \\
\text { overexpression }\end{array}$ & $\begin{array}{l}\text { GFR/urine protein/thrombocytopenia/ } \\
\text { neutropenia (primary), PFS (secondary) }\end{array}$ \\
\hline
\end{tabular}

1311, iodine 131; 177Lu, lutetium 177; 90Y, yttrium 90;CAPTEM, capecitabine plus temozolomide; CRS, cancer related symptoms; DCR, disease control rate; DOR, duration of response; G, grade; GEP NETs, gastroenteropancreatic neuroendocrine tumors;GFR, glomerular filtration rate; HOS, hormonal overproduction symptoms; MTD, maximum tolerated dose; NET-CUP, neuroendocrine tumor from carcinoma of unknown primary; ORR, objective response rate; PFS, progression-free survival; PS, performance status; RP2D, recommended phase II dose; SSTR, somatostatin receptor.

\section{Novel PRRT agents}

\section{LU-DOTA-EB-TATE}

One of the limitations of ${ }^{177} \mathrm{Lu}$-DOTATATE is the need to administer multiple doses to achieve anti-tumor effect with a tolerable toxicity profile for patients. Lutetium177-1,4,7,10-tetra-azacyclododecane-1,4,7,10-tetraacetic acid-Evans blue-Tyr3-octreotate (177Lu-DOTA-EB-TATE) represents a long-lasting SSA which may circumvent this necessity. A phase I study of ${ }^{177} \mathrm{Lu}$-DOTA-EB-TATE explored safety and dosimetry of a single dose of the drug (Wang et al. 2018). Of the eight patients treated, five received ${ }^{177} \mathrm{Lu}$-DOTA-EB-TATE while three received ${ }^{177} \mathrm{Lu}$-DOTATATE; all patients underwent serial wholebody planar and single-photon emission CT (SPECT)-CT scans. Compared to ${ }^{177} \mathrm{Lu}$-DOTATATE, patients treated with the investigational agent experienced extended blood circulation and a 7.9-fold increase in tumor dose delivery.

\section{Somatostatin-receptor antagonists}

In contrast to SSTR agonists, SSTR antagonists can occupy more binding sites with lower dissociation rates, leading to higher tumor uptake and shorter retention of radiation in healthy organs (Fani et al. 2017). ${ }^{177} \mathrm{Lu}-\mathrm{OPS} 201$ (also known as ${ }^{177} \mathrm{Lu}$ DOTA-JR11 or Satoreotide tetraxetan) is one of the antagonists that is now being tested in clinical trials (NCT02592707, NCT03773133). In vitro experiments comparing ${ }^{177} \mathrm{Lu}-\mathrm{OPS} 201$ to ${ }^{177} \mathrm{Lu}$-DOTATATE in osteosarcoma cells transfected with SSTR2 demonstrated that the former compound accumulates at cell membrane surfaces rather than being internalized, and elicits more dsDNA breaks (Dalm et al. 2016). In vivo experiments in $\mathrm{H} 69$ xenografts revealed that mice treated with ${ }^{177} \mathrm{Lu}$-OPS201 compared to ${ }^{177} \mathrm{Lu}$-DOTATATE had longer periods of tumor stabilization $(45 \pm 7$ days vs $41 \pm 2$ days) and longer median survivals (71 days vs 61 days). In a small pilot study utilizing a crossover design, four patients received treatment with both ${ }^{177} \mathrm{Lu}-\mathrm{OPS} 201$ and 177Lu-DOTATATE (Wild et al. 2014). Patients underwent whole-body imaging and SPECT/CT imaging posttreatment to measure biodistribution and underwent ${ }^{68} \mathrm{Ga}$-DOTATATE to assess response. Patients were found to have a tumor dose which was 1.7-10.6 times greater with the SSTR antagonist compared to with the SSTR agonist. These findings prompted a phase I study of ${ }^{177} \mathrm{Lu}-\mathrm{OPS} 201$ in 20 (45\% PNET) refractory well-differentiated NET patients (Reidy-Lagunes et al. 2019). Patients were treated with two cycles of the agent at 3-month intervals. Of these patients, six received one cycle while fourteen received two cycles. Response rates were 45\% (one complete response) and disease control was observed in $85 \%$ of patients. Median PFS was 21 months. Grade 4 myelosuppression was observed in 4 (57.1\%) patients after cycle 2 , necessitating a protocol amendment to limit (c) 2021 Society for Endocrinology Published by Bioscientifica Ltd. Printed in Great Britain 
total bone marrow exposure to one gray and reduce the cycle 2 dose by $50 \%$. The study remains ongoing with no further data updates as of yet.

\section{Alpha emitters}

With substantially higher linear energy and shorter penetration range, $\alpha$-emitters allow more precise targeted therapy with less scatter to surrounding tissue (Dodson et al. 2007). Bismuth-213, Actinium-225, and Lead-212 are three alpha emitters currently under evaluation in clinical trials (Table 2) (Miederer et al. 2008, Giesel et al. 2013). The activity of ${ }^{225}$ Ac-DOTATATE was reported in 32 patients with metastatic GEP NETs who attained stable or progressive disease after ${ }^{177} \mathrm{Lu}$-DOTATATE. Fifteen patients experienced PR while nine demonstrated SD. At a median follow up of 8 months, no patients experienced disease progression or death (Ballal et al. 2019). ${ }^{213} \mathrm{Bi}$-DOTATOC was evaluated in seven patients (six treated with intra-arterial and one with i.v. infusion) with progressive metastatic NETs refractory to $\beta$-emitting radiation. Chronic nephrotoxicity was moderate and hematologic toxicities were primarily grade 1 and 2; one patient, however, developed myelodysplastic syndrome with progression to acute leukemia (Kratochwil et al. 2014). The response rate achieved with the alpha emitter in patients already pretreated with ${ }^{177} \mathrm{Lu}$-DOTATATE is promising and suggests the agent class may be even more cytoreductive in PRRT-naïve patients. A dose escalation study with ${ }^{212} \mathrm{~Pb}$-DOTAMTATE is ongoing in PRRT-naïve NET patients [NCT03466216].

\section{Individualized dosimetry}

${ }^{177} \mathrm{Lu}$-DOTATATE is currently approved at a fixed dose of $7.4 \mathrm{GBq}$ per cycle for four cycles. Individualized dosimetry strategies, primarily focused at achieving a predefined renal exposure (conventionally $23 \mathrm{~Gy}$ ) or bone marrow exposure (2 Gy) have been proposed and tested (Sandstrom et al. 2015). Other potential advantages touted with individualized dosimetry include improving antitumor response through increasing radiation exposure to NET lesions (Cremonesi et al. 2018). These approaches however are limited by heterogeneity of dosimetric methodology and lack of uniformity with regards to tumor response assessment (Haug, 2020). In one study, such a strategy enabled $50 \%$ of patients to receive more than four cycles (>29.6 GBq) of ${ }^{177} \mathrm{Lu}$-DOTATATE (Del Prete et al. 2019). However, given the exceedingly low rates of nephrotoxicity observed with ${ }^{177} \mathrm{Lu}$-DOTATATE combined with prophylactic amino acids, it is unclear whether renal exposure represents a clinically relevant endpoint. Bone marrow exposure perhaps may be more clinically significant given that current calculations tend to be crude and likely underestimate risk of cytopenias and myelodysplasia.

\section{Conclusion}

PRRT has been an important advance for WD GEP NET patients. However, many questions remain regarding the optimal timing of treatment, the dosing strategy (fixed vs individualized), the selection of patients, which imaging studies and biomarkers are most useful pretreatment and on-treatment, and choice of radionuclide $\left({ }^{177} \mathrm{Lu}\right.$ alone or in combination with ${ }^{90} \mathrm{Y}$ ). Novel PRRT agents utilizing $\alpha$-emitters and SSTR antagonists are in clinical development and offer theoretical advantages over ${ }^{177} \mathrm{Lu}$-DOTATATE. It remains to be seen how these compounds will fare in larger randomized studies and ultimately compare against ${ }^{177} \mathrm{Lu}$-DOTATATE. Combination strategies to increase the potency of PRRT are also in development including ongoing trials combining the treatment with cytotoxic chemotherapy (CAPTEM and fluorouracil), radiation

Table 2 Studies with alpha emitting radionuclide-based PRRT.

\begin{tabular}{|c|c|c|c|c|c|c|}
\hline Radionuclide & Study type (disease tested) & $\begin{array}{l}\text { Number of } \\
\text { patients }\end{array}$ & Response rate & $\begin{array}{l}\text { Median } \\
\text { PFS or OS }\end{array}$ & $\begin{array}{l}\text { Notable adverse } \\
\text { events }\end{array}$ & Reference \\
\hline${ }^{225} \mathrm{AC}$ & $\begin{array}{l}\text { Prospective (GEP NETs pre- } \\
\text { treated with }{ }^{177} \text { Lu-DOTATATE) }\end{array}$ & 32 & $62.5 \%$ (all PR) & NR & $\begin{array}{l}\text { No G3/G4 } \\
\text { hematologic, renal } \\
\text { or hepatotoxicity }\end{array}$ & (Ballal et al. 2020) \\
\hline${ }^{213} \mathrm{Bi}$ & $\begin{array}{l}\text { Retrospective (GEP NET, bronchial } \\
\text { NET and prostate NET treated } \\
\text { with } 90 \text { Y/177Lu-DOTATOC) }\end{array}$ & 7 & $86 \%(1 \mathrm{CR})$ & NR & $\begin{array}{l}\text { one case of tMDS } \\
\text { and subsequently } \\
\text { AML }\end{array}$ & $\begin{array}{l}\text { (Kratochwil et al. } \\
\text { 2014) }\end{array}$ \\
\hline $212 \mathrm{~Pb}$ & Prospective (SSTR positive NETs) & 50 & NYR & NYR & NYR & (NCT03466216) \\
\hline
\end{tabular}

CR, complete response; G, grade; GEP NETs, gastroenteropancreatic neuroendocrine tumors; NYR, not yet recruiting; PR, partial response; SSTR, somatostatin receptor; tMDS, therapy related myelodysplastic syndrome.

(c) 2021 Society for Endocrinology Published by Bioscientifica Ltd. Printed in Great Britain 
sensitizers (triapine) and PARP inhibitors. PRRT will remain an important therapy for SSTR-expressing NET patients in the foreseeable future given the central role DNA damage inducing strategies play in the control of NETs, however the specific agents, modes of delivery and accompanying treatments may be quite different.

\section{Declaration of interest}

Dr Strosberg has consulted for Novartis. None of the other authors have conflicts to declare.

\section{Funding}

Dr Das's research efforts are funded by the grants P50CA236733 and 5K12CA90625-19.

\section{References}

Aljubran AH, Alrowaili M, Raef H, Bazarbashi S, Alzahrani AM, Almuhaideb A, Almanea H, Badran Aa, Al-Dalee A \& Tuli M 2019 Combination of everolimus and lu-177 PRRT in treatment of G1-2 neuroendocrine tumors (NET): Phase 1-2 study. Journal of Clinical Oncology 37 386-386. (https://doi.org/10.1200/JCO.2019.37.4_ suppl.386)

Ballal S, Yadav MP, Damle NA, Sahoo RK \& Bal C 2017 Concomitant 177Lu-DOTATATE and capecitabine therapy in patients with advanced neuroendocrine tumors: a long-term-outcome, toxicity, survival, and quality-of-life study. Clinical Nuclear Medicine $\mathbf{4 2}$ e457-e466. (https://doi.org/10.1097/RLU.0000000000001816)

Ballal S, Yadav MP, Bal C, Sahoo RK \& Tripathi M 2020 Broadening horizons with 225Ac-DOTATATE targeted alpha therapy for gastroenteropancreatic neuroendocrine tumour patients stable or refractory to 177Lu-DOTATATE PRRT: first clinical experience on the efficacy and safety. European Journal of Nuclear Medicine and Molecular Imaging 47 934-946. (https://doi.org/10.1007/s00259-019-04567-2)

Baum RP, Kulkarni HR, Singh A, Kaemmerer D, Mueller D, Prasad V, Hommann M, Robiller FC, Niepsch K, Franz H, et al. 2018 Results and adverse events of personalized peptide receptor radionuclide therapy with (90)Yttrium and (177)Lutetium in 1048 patients with neuroendocrine neoplasm. Oncotarget 9 16932-16950. (https://doi. org/10.18632/oncotarget.24524)

Bodei L, Kidd M, Paganelli G, Grana CM, Drozdov I, Cremonesi M, Lepensky C, Kwekkeboom DJ, Baum RP, Krenning EP, et al. 2015 Long-term tolerability of PRRT in 807 patients with neuroendocrine tumours: the value and limitations of clinical factors. European Journal of Nuclear Medicine and Molecular Imaging 42 5-19. (https:// doi.org/10.1007/s00259-014-2893-5)

Bodei L, Kidd MS, Singh A, Van Der Zwan WA, Severi S, Drozdov IA, Cwikla J, Baum RP, Kwekkeboom DJ, Paganelli G, et al. 2018 PRRT genomic signature in blood for prediction of (177)Lu-octreotate efficacy. European Journal of Nuclear Medicine and Molecular Imaging 45 1155-1169. (https://doi.org/10.1007/s00259-018-3967-6)

Braat AJAT, Kwekkeboom DJ, Kam BLR, Teunissen JJM, De Herder WW, Kma D, Van Rooij R, Krijger GC \& Jong Hwam DE 2018 Additional hepatic (166)Ho-radioembolization in patients with neuroendocrine tumours treated with (177)Lu-DOTATATE; a single center, interventional, non-randomized, non-comparative, open label, phase II study (HEPAR PLUS trial). BMC Gastroenterology 1884.
Brabander T, Van Der Zwan WA, Teunissen JJM, Kam BLR, Feelders RA, De Herder WW, Van Eijck CHJ, Franssen GJH, Krenning EP \& Kwekkeboom DJ 2017 Long-term efficacy, survival, and safety of. ((177)Lu-DOTA(0),Tyr(3)]octreotate in patients with gastroenteropancreatic and bronchial neuroendocrine tumors. Clinical Cancer Research 23 4617-4624. (https://doi. org/10.1158/1078-0432.CCR-16-2743)

Bushnell DL, O'Dorisio TM, O'Dorisio MS, Menda Y, Hicks RJ, Van Cutsem E, Baulieu JL, Borson-Chazot F, Anthony L, Benson AB, et al. 2010 90Y-edotreotide for metastatic carcinoid refractory to octreotide. Journal of Clinical Oncology 28 1652-1659. (https://doi. org/10.1200/JCO.2009.22.8585)

Cambiaghi V, Vitali E, Morone D, Peverelli E, Spada A, Mantovani G \& Lania AG 2017 Identification of human somatostatin receptor 2 domains involved in internalization and signaling in QGP-1 pancreatic neuroendocrine tumor cell line. Endocrine 56 146-157. (https://doi.org/10.1007/s12020-016-1026-2)

Chauhan A, Kohn E \& Del Rivero J 2019 Neuroendocrine tumors-less well known, often misunderstood, and rapidly growing in incidence. JAMA Oncology [epub]. (https://doi.org/10.1001/ jamaoncol.2019.4568)

Claringbold PG, Price RA \& Turner JH 2012 Phase I-II study of radiopeptide $177 \mathrm{Lu}$-octreotate in combination with capecitabine and temozolomide in advanced low-grade neuroendocrine tumors. Cancer Biotherapy and Radiopharmaceuticals 27 561-569. (https://doi. org/10.1089/cbr.2012.1276)

Coffey A 2019 Ga-68 dotatoc versus In-111 ocreotide. Journal of Nuclear Medicine 602038.

Cremonesi M, Ferrari ME, Bodei L, Chiesa C, Sarnelli A, Garibaldi C, Pacilio M, Strigari L, Summers PE, Orecchia R, et al. 2018 Correlation of dose with toxicity and tumour response to (90)Y- and (177) Lu-PRRT provides the basis for optimization through individualized treatment planning. European Journal of Nuclear Medicine and Molecular Imaging 45 2426-2441. (https://doi.org/10.1007/s00259018-4044-x)

Dalm SU, Nonnekens J, Doeswijk GN, De Blois E, Van Gent DC, Konijnenberg MW \& De Jong M 2016 Comparison of the therapeutic response to treatment with a 177Lu-labeled somatostatin receptor agonist and antagonist in preclinical models. Journal of Nuclear Medicine 57 260-265. (https://doi.org/10.2967/ jnumed.115.167007)

Dasari A, Shen C, Halperin D, Zhao B, Zhou S, Xu Y, Shih T \& Yao JC 2017 Trends in the incidence, prevalence, and survival outcomes in patients With neuroendocrine tumors in the United States. JAMA Oncology 3 1335-1342. (https://doi.org/10.1001/ jamaoncol.2017.0589)

de Jong M, Breeman WA, Valkema R, Bernard BF \& Krenning EP 2005 Combination radionuclide therapy using 177Lu- and 90Y-labeled somatostatin analogs. Journal of Nuclear Medicine 46 (Supplement 1) 13S-17S.

Del Prete M, Buteau FA, Arsenault F, Saighi N, Bouchard LO, Beaulieu A \& Beauregard JM 2019 Personalized (177)Lu-octreotate peptide receptor radionuclide therapy of neuroendocrine tumours: initial results from the P-PRRT trial. European Journal of Nuclear Medicine and Molecular Imaging 46 728-742. (https://doi.org/10.1007/s00259-0184209-7)

Delpassand ES, Samarghandi A, Zamanian S, Wolin EM, Hamiditabar M, Espenan GD, Erion JL, O'Dorisio TM, Kvols LK, Simon J, et al. 2014 Peptide receptor radionuclide therapy with 177Lu-DOTATATE for patients with somatostatin receptorexpressing neuroendocrine tumors: the first US phase 2 experience. Pancreas 43 518-525. (https://doi.org/10.1097/ MPA.0000000000000113)

Dodson H, Wheatley SP \& Morrison CG 2007 Involvement of centrosome amplification in radiation-induced mitotic catastrophe. Cell Cycle 6 364-370. (https://doi.org/10.4161/cc.6.3.3834) 
Ezziddin S, Attassi M, Yong-Hing CJ, Ahmadzadehfar H, Willinek W, Grunwald F, Guhlke S, Biersack HJ \& Sabet A 2014 Predictors of long-term outcome in patients with well-differentiated gastroenteropancreatic neuroendocrine tumors after peptide receptor radionuclide therapy with $177 \mathrm{Lu}$-octreotate. Journal of Nuclear Medicine 55 183-190. (https://doi.org/10.2967/jnumed.113.125336)

Fani M, Nicolas GP \& Wild D 2017 Somatostatin receptor antagonists for imaging and therapy. Journal of Nuclear Medicine 58 61S-66S. (https://doi.org/10.2967/jnumed.116.186783)

Forbes S, Baghdadi Y, Parkar F, Abraham T \& Love C 2019 Evaluating Ga-68 DOTATATE images: SUVmax vs Krenning score. Journal of Nuclear Medicine 601535.

Giesel FL, Wulfert S, Zechmann CM, Haberkorn U, Kratochwil C, Flechsig P, Kuder T, Schwartz LH \& Bruchertseifer F 2013 Contrastenhanced ultrasound monitoring of perfusion changes in hepatic neuroendocrine metastases after systemic versus selective arterial 177Lu/90Y-DOTATOC and 213Bi-DOTATOC radiopeptide therapy. Experimental Oncology 35 122-126.

Haug AR 2020 PRRT of neuroendocrine tumors: individualized dosimetry or fixed dose scheme? EJNMMI Research 10 35. (https:// doi.org/10.1186/s13550-020-00623-3)

Hicks RJ, Kwekkeboom DJ, Krenning E, Bodei L, Grozinsky-Glasberg S, Arnold R, Borbath I, Cwikla J, Toumpanakis C, Kaltsas G, et al. 2017 Enets consensus guidelines for the standards of care in neuroendocrine neoplasia: peptide receptor radionuclide therapy with radiolabeled somatostatin analogues. Neuroendocrinology $\mathbf{1 0 5}$ 295-309. (https://doi.org/10.1159/000475526)

Hofving T, Arvidsson Y, Almobarak B, Inge L, Pfragner R, Persson M, Stenman G, Kristiansson E, Johanson V \& Nilsson O 2018 The neuroendocrine phenotype, genomic profile and therapeutic sensitivity of GEPNET cell lines. Endocrine-Related Cancer 25 X1-X2. (https://doi.org/10.1530/ERC-17-0445e)

Hope TA, Abbott A, Colucci K, Bushnell DL, Gardner L, Graham WS, Lindsay S, Metz DC, Pryma DA, Stabin MG, et al. 2019 NANETS/ SNMMI procedure standard for somatostatin receptor-based peptide receptor radionuclide therapy with (177)Lu-DOTATATE. Journal of Nuclear Medicine 60 937-943. (https://doi.org/10.2967/ jnumed.118.230607)

Hope TA, Bodei L, Chan JA, El-Haddad G, Fidelman N, Kunz PL, Mailman J, Menda Y, Metz DC, Mittra ES, et al. 2020 NANETS/ SNMMI consensus statement on patient selection and appropriate use of (177)Lu-DOTATATE peptide receptor radionuclide therapy. Journal of Nuclear Medicine 61 222-227. (https://doi.org/10.2967/ jnumed.119.240911)

Imhof A, Brunner P, Marincek N, Briel M, Schindler C, Rasch H, Macke HR, Rochlitz C, Muller-Brand J \& Walter MA 2011 Response, survival, and long-term toxicity after therapy with the radiolabeled somatostatin analogue [90Y-DOTA]-TOC in metastasized neuroendocrine cancers. Journal of Clinical Oncology 29 2416-2423. (https://doi.org/10.1200/JCO.2010.33.7873)

Kanakis G, Grimelius L, Spathis A, Tringidou R, Rassidakis GZ, Oberg K, Kaltsas G \& Tsolakis AV 2015 Expression of somatostatin receptors 1-5 and dopamine receptor 2 in lung carcinoids: implications for a therapeutic role. Neuroendocrinology 101 211-222. (https://doi. org/10.1159/000381061)

Kesavan M, Claringbold PG \& Turner JH 2014 Hematological toxicity of combined 177Lu-octreotate radiopeptide chemotherapy of gastroenteropancreatic neuroendocrine tumors in long-term follow-up. Neuroendocrinology 99 108-117. (https://doi. org/10.1159/000362558)

Kratochwil C, Giesel FL, Bruchertseifer F, Mier W, Apostolidis C, Boll R, Murphy K, Haberkorn U \& Morgenstern A $2014{ }^{213}$ Bi-DOTATOC receptor-targeted alpha-radionuclide therapy induces remission in neuroendocrine tumours refractory to beta radiation: a first-inhuman experience. European Journal of Nuclear Medicine and Molecular Imaging 2 2106-2119. (https://doi.org/10.1007/s00259-014-2857-9)
Kratochwil C, Stefanova M, Mavriopoulou E, Holland-Letz T, Dimitrakopoulou-Strauss A, Afshar-Oromieh A, Mier W, Haberkorn U \& Giesel FL 2015 SUV of [68Ga]DOTATOC-PET/CT predicts response probability of PRRT in neuroendocrine tumors. Molecular Imaging and Biology 17 313-318. (https://doi.org/10.1007/ s11307-014-0795-3)

Krenning EP, Bakker WH, Breeman WA, Koper JW, Kooij PP, Ausema L, Lameris JS, Reubi JC \& Lamberts SW 1989 Localisation of endocrinerelated tumours with radioiodinated analogue of somatostatin. Lancet 1 242-244. (https://doi.org/10.1016/s0140-6736(89)91258-0)

Krenning EP, Kwekkeboom DJ, Oei HY, De Jong RJ, Dop FJ, Reubi JC \& Lamberts SW 1994 Somatostatin-receptor scintigraphy in gastroenteropancreatic tumors. An overview of European results. Annals of the New York Academy of Sciences 733 416-424. (https://doi. org/10.1111/j.1749-6632.1994.tb17291.x)

Kunikowska J, Krolicki L, Hubalewska-Dydejczyk A, Mikolajczak R, SowaStaszczak A \& Pawlak D 2011 Clinical results of radionuclide therapy of neuroendocrine tumours with 90Y-DOTATATE and tandem 90Y/177Lu-DOTATATE: which is a better therapy option? European Journal of Nuclear Medicine and Molecular Imaging 38 1788-1797. (https://doi.org/10.1007/s00259-011-1833-x)

Kunikowska J, Pawlak D, Bak MI, Kos-Kudla B, Mikolajczak R \& Krolicki L 2017 Long-term results and tolerability of tandem peptide receptor radionuclide therapy with (90)Y/(177)Lu-DOTATATE in neuroendocrine tumors with respect to the primary location: a 10-year study. Annals of Nuclear Medicine 31 347-356. (https://doi. org/10.1007/s12149-017-1163-6)

Kwekkeboom DJ, Bakker WH, Kam BL, Teunissen JJ, Kooij PP, De Herder WW, Feelders RA, Van Eijck CH, De Jong M, Srinivasan A, et al. 2003 Treatment of patients with gastro-entero-pancreatic (GEP) tumours with the novel radiolabelled somatostatin analogue. (177Lu-DOTA(0),Tyr3]octreotate. European Journal of Nuclear Medicine and Molecular Imaging 30 417-422. (https://doi.org/10.1007/s00259002-1050-8)

Lundsten S, Spiegelberg D, Raval NR \& Nestor M 2020 The radiosensitizer Onalespib increases complete remission in (177) Lu-DOTATATE-treated mice bearing neuroendocrine tumor xenografts. European Journal of Nuclear Medicine and Molecular Imaging 47 980-990. (https://doi.org/10.1007/s00259-019-04673-1)

Merola E, Prasad V, Pascher A, Pape UF, arsenic R, denecke T, fehrenbach U, wiedenmann B \& pavel ME 2020 Peritoneal carcinomatosis in gastro-entero-pancreatic neuroendocrine neoplasms: clinical impact and effectiveness of the available therapeutic options. Neuroendocrinology 110 517-524. (https://doi. org/10.1159/000503144)

Miederer M, Henriksen G, Alke A, Mossbrugger I, QuintanillaMartinez L, Senekowitsch-Schmidtke R \& Essler M 2008 Preclinical evaluation of the alpha-particle generator nuclide $225 \mathrm{Ac}$ for somatostatin receptor radiotherapy of neuroendocrine tumors. Clinical Cancer Research 14 3555-3561. (https://doi. org/10.1158/1078-0432.CCR-07-4647)

Naraev BG, Ramirez RA, Kendi AT \& Halfdanarson TR 2019 Peptide receptor radionuclide therapy for patients With advanced lung carcinoids. Clinical Lung Cancer 20 e376-e392. (https://doi. org/10.1016/j.cllc.2019.02.007)

Nonnekens J, Van Kranenburg M, Beerens CE, Suker M, Doukas M, Van Eijck CH, De Jong M \& Van Gent DC 2016 Potentiation of peptide receptor radionuclide therapy by the PARP inhibitor olaparib. Theranostics 6 1821-1832. (https://doi.org/10.7150/thno.15311)

Oronsky B, Ma PC, Morgensztern D \& Carter CA 2017 Nothing but NET: a review of neuroendocrine tumors and carcinomas. Neoplasia 19 991-1002. (https://doi.org/10.1016/j.neo.2017.09.002)

Otte A, Herrmann R, Heppeler A, Behe M, Jermann E, Powell P, Maecke HR \& Muller J 1999 Yttrium-90 DOTATOC: first clinical results. European Journal of Nuclear Medicine 26 1439-1447. (https:// doi.org/10.1007/s002590050476) (c) 2021 Society for Endocrinology Published by Bioscientifica Ltd. Printed in Great Britain 
Pach D, Sowa-Staszczak A, Kunikowska J, Krolicki L, Trofimiuk M, Stefanska A, Tomaszuk M, Glowa B, Mikolajczak R, Pawlak D, et al. 2012 Repeated cycles of peptide receptor radionuclide therapy (PRRT) - results and side-effects of the radioisotope 90Y-DOTA TATE, 177Lu-DOTA TATE or 90Y/177Lu-DOTA TATE therapy in patients with disseminated NET. Radiotherapy and Oncology 102 45-50. (https://doi.org/10.1016/j.radonc.2011.08.006)

Pavlakis N, Ransom DT, Wyld D, Sjoquist KM, Asher R, Gebski V, Wilson K, Kiberu AD, Burge ME, Macdonald W, et al. 2020 First results for Australasian Gastrointestinal Trials Group (AGITG) control net study: phase II study of 177Lu-octreotate peptide receptor radionuclide therapy (LuTate PRRT) +/- capecitabine, temozolomide (CAPTEM) for midgut neuroendocrine tumors (mNETs). Journal of Clinical Oncology 38 604-604. (https://doi.org/10.1200/JCO.2020.38.4_suppl.604)

Purohit NK, Shah RG, Adant S, Hoepfner M, Shah GM \& Beauregard JM 2018 Potentiation of (177)Lu-octreotate peptide receptor radionuclide therapy of human neuroendocrine tumor cells by PARP inhibitor. Oncotarget 9 24693-24706. (https://doi.org/10.18632/ oncotarget.25266)

Reidy-Lagunes D, Pandit-Taskar N, O'donoghue JA, Krebs S, Staton KD, Lyashchenko SK, Lewis JS, Raj N, Gonen M, Lohrmann C, et al. 2019 Phase I trial of well-differentiated neuroendocrine tumors (NETs) with radiolabeled somatostatin antagonist (177)Lu-Satoreotide tetraxetan. Clinical Cancer Research 25 6939-6947. (https://doi. org/10.1158/1078-0432.CCR-19-1026)

Remes SM, Leijon HL, Vesterinen TJ, Arola JT \& Haglund CH 2019 Immunohistochemical expression of somatostatin receptor subtypes in a panel of neuroendocrine neoplasias. Journal of Histochemistry and Cytochemistry 67 735-743. (https://doi. org/10.1369/0022155419856900)

Riff BP, Yang YX, Soulen MC, Pryma DA, Bennett B, Wild D, Nicolas G, Teitelbaum UR \& Metz DC 2015 Peptide receptor radionuclide therapy-induced hepatotoxicity in patients with metastatic neuroendocrine tumors. Clinical Nuclear Medicine 40 845-850. (https://doi.org/10.1097/RLU.0000000000000935)

Sabet A, Biersack HJ \& Ezziddin S 2016 Advances in peptide receptor radionuclide therapy. Seminars in Nuclear Medicine 46 40-46. (https:// doi.org/10.1053/j.semnuclmed.2015.09.005)

Sandstrom M, Ilan E, Karlberg A, Johansson S, Freedman N \& GarskeRoman U 2015 Method dependence, observer variability and kidney volumes in radiation dosimetry of (177)Lu-DOTATATE therapy in patients with neuroendocrine tumours. EJNMMI Physics 224 (https://doi.org/10.1186/s40658-015-0127-y)

Seregni E, Maccauro M, Chiesa C, Mariani L, Pascali C, Mazzaferro V, De Braud F, Buzzoni R, Milione M, Lorenzoni A, et al. 2014 Treatment with tandem [90Y]DOTA-TATE and [177Lu]DOTA-TATE of neuroendocrine tumours refractory to conventional therapy. European Journal of Nuclear Medicine and Molecular Imaging $\mathbf{4 1}$ 223-230. (https://doi.org/10.1007/s00259-013-2578-5)

Singh A, Zhang J, Kulkarni HR \& Baum RP 2019 Intra-arterial PRRT of sstr-expressing tumors in patients with hepatic only versus extrahepatic tumor: efficacy and safety evaluation. Journal of Nuclear Medicine 60625.

Sorbye H, Kong G \& Grozinsky-Glasberg S 2020 PRRT in high-grade gastroenteropancreatic neuroendocrine neoplasms (WHO G3). Endocrine-Related Cancer 27 R67-R77. (https://doi.org/10.1530/ERC19-0400)

Spetz J, Langen B, Rudqvist N, Parris TZ, Helou K, Nilsson O \& ForssellAronsson E 2017 Hedgehog inhibitor sonidegib potentiates (177) Lu-octreotate therapy of GOT1 human small intestine neuroendocrine tumors in nude mice. BMC Cancer 17 528. (https:// doi.org/10.1186/s12885-017-3524-x)

Strosberg J, El-Haddad G, Wolin E, Hendifar A, Yao J, Chasen B, Mittra E, Kunz PL, Kulke MH, Jacene H, et al. 2017 Phase 3 trial of (177)Lu-Dotatate for midgut neuroendocrine tumors. New England
Journal of Medicine 376 125-135. (https://doi.org/10.1056/ NEJMoa1607427)

Strosberg J, Wolin E, Chasen B, Kulke M, Bushnell D, Caplin M, Baum RP, Kunz P, Hobday T, Hendifar A, et al. 2018a Health-related quality of life in patients with progressive midgut neuroendocrine tumors treated with (177)Lu-dotatate in the Phase III NETTER-1 trial. Journal of Clinical Oncology 36 2578-2584. (https://doi.org/10.1200/ JCO.2018.78.5865)

Strosberg JR, Wolin EM, Chasen BA, Kulke MH, Bushnell DL, Caplin ME, Baum RP, Hobday TJ, Hendifar AE, Ravasi L, et al. 2018b Clinical outcomes in patients with baseline renal dysfunction in the NETTER-1 study: 177Lu-Dotatate vs. high dose octreotide in progressive midgut neuroendocrine tumors. Journal of Clinical Oncology 36 4102-4102. (https://doi.org/10.1200/JCO.2018.36.15_ suppl.4102)

Strosberg J, Kunz PL, Hendifar A, Yao J, Bushnell D, Kulke MH, Baum RP, Caplin M, Ruszniewski P, Delpassand E, et al. 2020a Impact of liver tumour burden, alkaline phosphatase elevation, and target lesion size on treatment outcomes with (177)Lu-Dotatate: an analysis of the NETTER-1 study. European Journal of Nuclear Medicine and Molecular Imaging 47 2372-2382. (https://doi.org/10.1007/s00259. 020-04709-x)

Strosberg JR, Al-Toubah T, Pelle E, Smith J, Haider M, Hutchinson T, Fleming JB \& El-Haddad G $2020 b$ Risk of bowel obstruction in patients with mesenteric/peritoneal disease receiving peptide receptor radionuclide therapy (PRRT). Journal of Nuclear Medicine 62 69-72.

Valkema R, De Jong M, Bakker WH, Breeman WA, Kooij PP, Lugtenburg PJ, De Jong FH, Christiansen A, Kam BL, De Herder WW, et al. 2002 Phase I study of peptide receptor radionuclide therapy with [In-DTPA] octreotide: the Rotterdam experience. Seminars in Nuclear Medicine 32 110-122. (https://doi.org/10.1053/ snuc/2002.31025)

van der Zwan WA, Brabander T, Kam BLR, Teunissen JJM, Feelders RA, Hofland J, Krenning EP \& De Herder WW 2019 Salvage peptide receptor radionuclide therapy with. ((177)Lu-DOTA,Tyr(3)]octreotate in patients with bronchial and gastroenteropancreatic neuroendocrine tumours. European Journal of Nuclear Medicine and Molecular Imaging 46 704-717. (https://doi.org/10.1007/s00259-0184158-1)

van Essen M, Krenning EP, Kam BL, De Herder WW, Van Aken MO \& Kwekkeboom DJ 2008 Report on short-term side effects of treatments with $177 \mathrm{Lu}$-octreotate in combination with capecitabine in seven patients with gastroenteropancreatic neuroendocrine tumours. European Journal of Nuclear Medicine and Molecular Imaging 35 743-748. (https://doi.org/10.1007/s00259-007-0688-7)

Vinjamuri S, Gilbert TM, Banks M, Mckane G, Maltby P, Poston G, Weissman H, Palmer DH, Vora J, Pritchard DM, et al. 2013 Peptide receptor radionuclide therapy with (90)Y-DOTATATE/(90) Y-DOTATOC in patients with progressive metastatic neuroendocrine tumours: assessment of response, survival and toxicity. British Journal of Cancer 108 1440-1448. (https://doi.org/10.1038/ bjc.2013.103)

Wang H, Cheng Y, Zhang J, Zang J, Li H, Liu Q, Wang J, Jacobson O, Li F, Zhu Z, et al. 2018 Response to single low-dose 177Lu-DOTA-EBTATE treatment in patients with advanced neuroendocrine neoplasm: a Prospective Pilot Study. Theranostics 8 3308-3316. (https://doi.org/10.7150/thno.25919)

Wild D, Fani M, Fischer R, Del Pozzo L, Kaul F, Krebs S, Fischer R, Rivier JE, Reubi JC, Maecke HR, et al. 2014 Comparison of somatostatin receptor agonist and antagonist for peptide receptor radionuclide therapy: a pilot study. Journal of Nuclear Medicine $\mathbf{5 5}$ 1248-1252. (https://doi.org/10.2967/jnumed.114.138834)

Yamada Y, Post SR, Wang K, Tager HS, Bell GI \& Seino S 1992 Cloning and functional characterization of a family of human and mouse https://erc bioscientifica com

https://doi.org/10.1530/ERC-20-0360 c) 2021 Society for Endocrinology Published by Bioscientifica Ltd. Printed in Great Britain 
somatostatin receptors expressed in brain, gastrointestinal tract, and kidney. PNAS 89 251-255. (https://doi.org/10.1073/ pnas.89.1.251)

Yordanova A, Ahrens H, Feldmann G, Brossart P, Gaertner FC, Fottner C, Weber MM, Ahmadzadehfar H, Schreckenberger M, Miederer M, et al. 2019 Peptide receptor radionuclide therapy combined with chemotherapy in patients with neuroendocrine tumors. Clinical
Nuclear Medicine 44 e329-e335. (https://doi.org/10.1097/ RLU.0000000000002532)

Zhang J, Kulkarni HR, Singh A, Niepsch K, Muller D \& Baum RP 2019 Peptide receptor radionuclide therapy in Grade 3 neuroendocrine neoplasms: safety and survival analysis in 69 patients. Journal of Nuclear Medicine 60 377-385. (https://doi.org/10.2967/ jnumed.118.215848)

Received in final form 8 February 2021

Accepted 10 February 2021

Accepted Manuscript published online 19 February 2021 (c) 2021 Society for Endocrinology Published by Bioscientifica Ltd Printed in Great Britain 